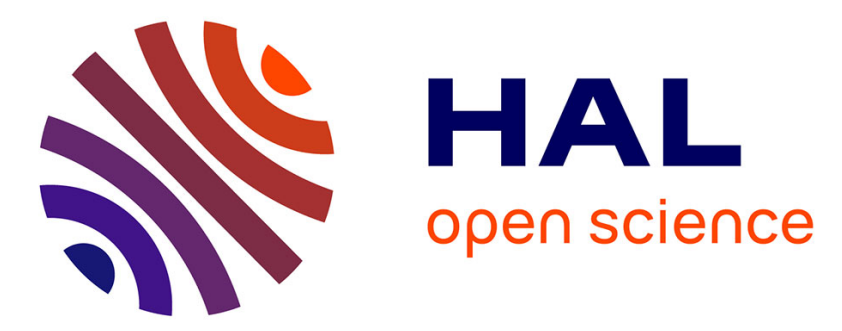

\title{
A discrete-time control strategy for dynamic walking of a planar under-actuated biped robot
}

\author{
Ahmed Chemori
}

\section{To cite this version:}

Ahmed Chemori. A discrete-time control strategy for dynamic walking of a planar under-actuated biped robot. IROS: Intelligent Robots and Systems, Oct 2009, St. Louis, MO, United States. pp.32263231, 10.1109/IROS.2009.5353965 . lirmm-00429803

\section{HAL Id: lirmm-00429803 https://hal-lirmm.ccsd.cnrs.fr/lirmm-00429803}

Submitted on 4 Nov 2009

HAL is a multi-disciplinary open access archive for the deposit and dissemination of scientific research documents, whether they are published or not. The documents may come from teaching and research institutions in France or abroad, or from public or private research centers.
L'archive ouverte pluridisciplinaire HAL, est destinée au dépôt et à la diffusion de documents scientifiques de niveau recherche, publiés ou non, émanant des établissements d'enseignement et de recherche français ou étrangers, des laboratoires publics ou privés. 


\title{
A discrete-time control strategy for dynamic walking of a planar under-actuated biped robot
}

\author{
Ahmed Chemori
}

\begin{abstract}
This paper deals with a discret-time control approach, proposed for the control of a five-link, four-actuator planar biped walker. The approach is based on the choice of a particular class of walk configurations that enables a full state controllability avoiding the need to use Poincaré-like argumentation in the proof of motion's reproducibility (stability of limit cycles). Simulation results attest the efficiency of the proposed control approach.
\end{abstract}

\section{INTRODUCTION}

In last the decades, the robotics community has shown increasing interest in the area of legged walking robots [14], [1]. An excellent database of biped walking robots built all over the world can be found in [1]. One of the serious reasons for exploring the use of walking robots relies in the fact that they can co-exist and collaborate with their creators without any costly modification to the environment created for humans.

Currently, many research groups over the world are working on biped robots, either on optimization of leg and foot trajectory, stable walking control, or hardware design. The main thrust of current research on biped control includes many proposed control approaches, such as passivity based control [16], robust sliding mode control [3], impedance control [12], nonlinear predictive control [4], optimal control [8], Lyapunov based control [5] computed torque control [2], tracking control [15], intuitive control [13], intelligent learning control [11], and neural network control [10].

From a control point of view, the major interest of biped robots comes from the following difficulties that are inherent to control of such systems:

1) biped robots have generally high degrees of freedom mechanical structure, therefore many links and joints are to be coordinated to achieve walking gaits,

2) dynamics of biped robots includes high nonlinearities,

3) their hybrid nature resulting from impacts with the ground producing discontinuities (jumps) in the generalized velocities,

4) another interesting point is under-actuation. Biped walking robots may be under-actuated (case of our robot Rabbit) that is the number of actuators is fewer than the number of degrees of freedom (d.o.f),

5) the variable structure resulting from the switching between different walking phases.

In this paper a new control strategy is proposed to control a five-link, four-actuator biped walking robot. The directly

A. Chemori is with LIRMM, Univ. Montpellier 2 - CNRS, 161 rue Ada, 34392 Montpellier, France chemori@lirmm. fr controlled d.o.f are the absolute angular positions of the lower-limbs of the robot $X_{c}=\left(\pi-q_{31}, \pi-q_{32}, q_{41}, q_{42}\right)^{T}$, whereas the indirectly controlled d.o.f is the position of the torso defined by $X_{n c}=q_{1}$ (unactuated coordinate). The main idea consists in the choice of particular configurations, for which the dynamics of the system is linearized. Then a discret-time feedback is proposed to control the whole system to produce stable cyclic walking gaits.

This paper is organized as follows. The first section is this introduction. In section II, the biped robot prototype is described, and its dynamical model is discussed. Section III is devoted to the unactuated coordinate dynamics. The proposed control strategy is detailed in section IV. Numerical simulations are given in section $\mathrm{V}$ to illustrate the efficiency of the proposed control strategy. Finally, concluding remarks are drawn in section VI.

\section{ROBOT DESCRIPTION AND DYNAMICS}

Illustrated by the schematic view on figure 1, RABBIT has two legs and a torso. It has the original specificity enabling both walking and running gaits [6]. Thanks to a guidance device, the robot is laterally stabilized, then its motions can be considered in the sagittal plane.

The mechanical structure of the robot is illustrated on figure 2 showing its generalized coordinates and control inputs. The

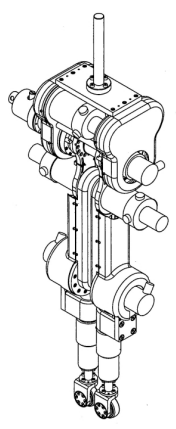

Fig. 1. Schematic view of the biped RABBIT

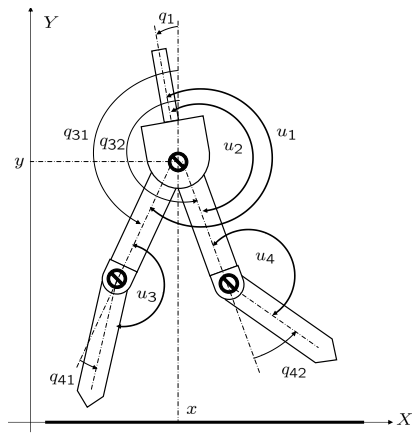

Fig. 2. RABBIT's mechanical structure use of Lagrange formulation [17] enables the computation of the nonlinear dynamic model of the robot moving in the sagittal plane without contact with the ground:

$$
M(q) \ddot{q}+N(q, \dot{q}) \dot{q}+G(q)=S u
$$

where $M(q) \in \mathbb{R}^{7 \times 7}$ is the inertia matrix, $N(q, \dot{q}) \in \mathbb{R}^{7 \times 7}$ is the matrix of the centrifugal and Coriolis forces, $G(q) \in \mathbb{R}^{7}$ 
is the vector of gravitational forces, $u \in \mathbb{R}^{4}$ is the vector of control inputs, $S \in \mathbb{R}^{7 \times 4}$ is a torque distribution matrix, $q=\left(\begin{array}{lllllll}q_{31} & q_{41} & q_{32} & q_{42} & q_{1} & x & y\end{array}\right)^{T} \in \mathbb{R}^{7}$ is the vector of generalized coordinates of the robot (cf. figure 2). The walking cycle of the biped can be decomposed into three walking phases, namely the swing phase (or single support phase), the impact phase and the double support phase. In the case of this study the double support phase is assumed to be instantaneous as in [7], [4] and others. The control of the biped robot on the whole walking cycle needs the computation of its dynamic model on the different walking phases. This is the subject of forthcoming sections.

\section{A. The swing phase model}

The dynamic model describing the biped robot in the swing phase is given by the following:

$$
\left\{\begin{array}{l}
M(q) \ddot{q}+N(q, \dot{q}) \dot{q}+G(q)=S u+J_{1}^{T}(q) \lambda \\
J_{1}(q) \ddot{q}+\frac{\partial^{2} \Phi_{1}}{\partial q^{2}} \dot{q}^{2}=0
\end{array}\right.
$$

where $\lambda$ is the Lagrange multipliers of the contact forces, $\Phi_{1}(q)$ is the function of the contact constraints, and $J_{1}(q)$ is their Jacobian matrix.

\section{B. The impact phase model}

Under the assumption that the swing foot and the ground are rigid bodies, the end of the swing phase is characterized by a collision between the swing foot and the ground [18]. The application of the non-regular mechanics laws enables us to deduce the dynamic model of the rigid impact that happens between the swing-leg foot and the ground [9]:

$$
\left\{\begin{array}{l}
\dot{q}^{+}=\left[I-M^{-1} J_{2}^{T}\left(J_{2} M^{-1} J_{2}^{T}\right)^{-1} J_{2}\right] \dot{q}^{-}=D(q) \dot{q}^{-} \\
\lambda=-\left[\left(J_{2} M^{-1} J_{2}^{T}\right)^{-1} J_{2}\right] \dot{q}^{-}
\end{array}\right.
$$

It gives $\dot{q}^{+}$and $\lambda$ as functions of the pre-impact positions $q^{-}$and velocities $\dot{q}^{-}$. This dynamics may be expressed as:

$$
\left(\begin{array}{c}
q^{+} \\
\dot{q}^{+}
\end{array}\right)=\Delta(q)\left(\begin{array}{c}
q^{-} \\
\dot{q}^{-}
\end{array}\right)
$$

where the matrix $\Delta(q)$ includes also the relabeling of the legs (i.e the stance leg becomes a swing leg and vice versa).

\section{DYNAMICS OF THE TORSO}

Let us consider the dynamic model (1) of the robot. Extracted from it, the dynamics of the torso is given by:

$$
\begin{aligned}
\left(\frac{1}{4} m_{1} l_{1}^{2}+I_{1}\right) \ddot{q}_{1} & =\frac{1}{2} m_{1} l_{1} \cos \left(q_{1}\right) \ddot{x}+ \\
& \frac{1}{2} m_{1} l_{1} \sin \left(q_{1}\right)(\ddot{y}+g)-u_{1}-u_{2}
\end{aligned}
$$

where $u_{1}$ and $u_{2}$ are the control inputs of articulations $q_{31}$ and $q_{32}$ respectively, whose dynamics is given by:

$$
\begin{aligned}
& M_{1}(q)\left(\begin{array}{l}
\ddot{q}_{31} \\
\ddot{q}_{32}
\end{array}\right)+M_{2}(q)\left(\begin{array}{l}
\ddot{q}_{41} \\
\ddot{q}_{42}
\end{array}\right)+M_{3}(q)\left(\begin{array}{l}
\ddot{x} \\
\ddot{y}
\end{array}\right)+ \\
& +\bar{N}_{1,3}(q, \dot{q}) \dot{q}+\bar{G}_{1,3}(q)=C(q)\left(\begin{array}{l}
\lambda_{n} \\
\lambda_{t}
\end{array}\right)+\left(\begin{array}{l}
u_{1} \\
u_{2}
\end{array}\right)
\end{aligned}
$$

with $M_{1}:=\left(\begin{array}{ll}m_{11} & m_{13} \\ m_{31} & m_{33}\end{array}\right) \in \mathbb{R}^{2} ; M_{2}:=\left(\begin{array}{ll}m_{12} & m_{14} \\ m_{32} & m_{34}\end{array}\right) \in \mathbb{R}^{2}$

$M_{3}:=\left(\begin{array}{ll}m_{16} & m_{17} \\ m_{36} & m_{37}\end{array}\right) \in \mathbb{R}^{2} ; C(q):=\left(\begin{array}{ll}J_{1}^{T}(1,1) & J_{1}^{T}(1,2) \\ J_{1}^{T}(3,1) & J_{1}^{T}(3,2)\end{array}\right)$

now if we restrict ourselves to trajectories for which:

- the following angles:

$$
\begin{gathered}
\left|\pi-q_{31}\right| ;\left|\pi-q_{32}\right| \quad ; \quad q_{41} \quad ; \quad q_{42} \\
\left|\pi-\left(q_{32}+q_{42}\right)\right| \quad ; \quad\left|\pi-\left(q_{31}+q_{41}\right)\right|
\end{gathered}
$$

can be considered as 'small angles',

- the amplitudes of the time derivatives of the angles are of the same order of magnitude of the angles themselves,

then it is clear that equation (6) can be greatly simplified by keeping only the first order terms to obtain:

$$
\begin{aligned}
& M_{1}\left(\begin{array}{l}
\ddot{q}_{31} \\
\ddot{q}_{32}
\end{array}\right)+M_{2}\left(\begin{array}{l}
\ddot{q}_{41} \\
\ddot{q}_{42}
\end{array}\right)+M_{3}\left(\begin{array}{l}
\ddot{x} \\
\ddot{y}
\end{array}\right)+G_{0}\left(\begin{array}{c}
\pi-q_{31} \\
\pi-q_{32} \\
q_{41} \\
q_{42}
\end{array}\right)= \\
& C(q)\left(\begin{array}{l}
\lambda_{n} \\
\lambda_{t}
\end{array}\right)+\left(\begin{array}{l}
u_{1} \\
u_{2}
\end{array}\right)
\end{aligned}
$$

Note that matrices $M_{1}, M_{2}$ and $M_{3}$ are now constant matrices, there is no more Coriolis term and the gravity term is affine in the directly controlled variables $q_{31}, q_{41}, q_{32}$ and $q_{42}$ with $G_{0}:=\left[\operatorname{Diag}\left(-g_{34},-g_{34}\right) \operatorname{Diag}\left(g_{4}, g_{4}\right)\right]$, and $g_{34}:=\frac{g}{2}\left(m_{3} l_{3}+m_{4}\left(2 l_{3}+l_{4}\right)\right) ; g_{4}=\frac{g}{2} m_{4} l_{4}$

To go further, the approximate expressions of $\lambda_{n}$ and $\lambda_{t}$ have to be investigated. For that, let us have a look on the $x$ and $y$ equations. Under the above assumptions, the equations for $x$ and $y$ can be written in the following simplified form:

$$
\begin{aligned}
& m \ddot{x}=\lambda_{t}-\left(\begin{array}{llll}
\frac{g_{34}}{g} & \frac{g_{34}}{g} & \frac{g_{4}}{g} & \frac{g_{4}}{g}
\end{array}\right)\left(\begin{array}{l}
\ddot{q}_{31} \\
\ddot{q}_{32} \\
\ddot{q}_{41} \\
\ddot{q}_{42}
\end{array}\right)+\left(\frac{1}{2} m_{1} l_{1}\right) \ddot{q}_{1} \\
& m \ddot{y}=-m g+\lambda_{n}
\end{aligned}
$$

using equations (9)-(10) to express $C(q)\left(\begin{array}{c}\lambda_{n} \\ \lambda_{t}\end{array}\right)$ appearing in (8), leads after some manipulations to:

$$
\begin{gathered}
M_{1}\left(\begin{array}{c}
\ddot{q}_{31} \\
\ddot{q}_{32}
\end{array}\right)+M_{2}\left(\begin{array}{c}
\ddot{q}_{41} \\
\ddot{q}_{42}
\end{array}\right)-G_{2}\left(\begin{array}{l}
\ddot{q}_{31} \\
\ddot{q}_{32} \\
\ddot{q}_{41} \\
\ddot{q}_{42}
\end{array}\right)+\left(M_{3}-M_{4}\right)\left(\begin{array}{l}
\ddot{x} \\
\ddot{y}
\end{array}\right)+ \\
\left(G_{0}-C_{1}\right)\left(\begin{array}{c}
\pi-q_{31} \\
\pi-q_{32} \\
q_{41} \\
q_{42}
\end{array}\right)-\left(\begin{array}{c}
\frac{1}{2} m_{1} l_{1}\left(l_{3}+l_{4}\right) \\
0
\end{array}\right) \ddot{q}_{1}=\left(\begin{array}{l}
u_{1} \\
u_{2}
\end{array}\right)
\end{gathered}
$$

Now using geometric arguments (first order approximation) to write $\ddot{x}$ and $\ddot{y}$ under swing phase conditions gives:

$$
\left(\begin{array}{l}
\ddot{x} \\
\ddot{y}
\end{array}\right) \approx\left(\begin{array}{cc}
-\left(l_{3}+l_{4}\right) & -l_{4} \\
0 & 0
\end{array}\right)\left(\begin{array}{l}
\ddot{q}_{31} \\
\ddot{q}_{41}
\end{array}\right)
$$

that gives when injected in (11):

$$
\begin{aligned}
& {\left[M_{3}^{*}+\left(\begin{array}{ll}
M_{1} & M_{2}
\end{array}\right)-G_{2}\right]\left(\begin{array}{l}
\ddot{q}_{31} \\
\ddot{q}_{32} \\
\ddot{q}_{41} \\
\ddot{q}_{42}
\end{array}\right)+\left(G_{0}-C_{1}\right)\left(\begin{array}{c}
\pi-q_{31} \\
\pi-q_{32} \\
q_{41} \\
q_{42}
\end{array}\right)-} \\
& \left(\begin{array}{c}
\frac{1}{2} m_{1} l_{1}\left(l_{3}+l_{4}\right) \\
0
\end{array}\right) \ddot{q}_{1}=\left(\begin{array}{l}
u_{1} \\
u_{2}
\end{array}\right)
\end{aligned}
$$


with $M_{3}^{*}:=\left(M_{3}-M_{4}\right)\left(\begin{array}{cccc}-\left(l_{3}+l_{4}\right) & 0 & -l_{4} & 0 \\ 0 & 0 & 0 & 0\end{array}\right)$

Now, equation (13) may be written in terms of $X_{c}$ (introduced in section I) and $\ddot{X}_{c}$ as follows:

$$
\begin{aligned}
& {\left[M_{3}^{*}+\left(\begin{array}{ll}
M_{1} & M_{2}
\end{array}\right)-G_{2}\right]\left(\begin{array}{cc}
-\mathbb{I} & 0 \\
0 & \mathbb{I}
\end{array}\right) \ddot{X}_{c}+\left(G_{0}-C_{1}\right) X_{c} } \\
- & \left(\begin{array}{c}
\frac{1}{2} m_{1} l_{1}\left(l_{3}+l_{4}\right) \\
0
\end{array}\right) \ddot{q}_{1}=\left(\begin{array}{l}
u_{1} \\
u_{2}
\end{array}\right)
\end{aligned}
$$

where $\mathbb{I}$ in (14) represents the identity matrix in $\mathbb{R}^{2 \times 2}$. Finally, by using (14), one may write $u_{1}+u_{2}$ in terms of $X_{c}$ and its second derivative as:

$$
u_{1}+u_{2}=L_{0}^{*} X_{c}+L_{2}^{*} \ddot{X}_{c}-\frac{1}{2} m_{1} l_{1}\left(l_{3}+l_{4}\right) \ddot{q}_{1}
$$

where

$L_{0}^{*}=\left(\begin{array}{ll}1 & 1\end{array}\right)\left(G_{0}-C_{1}\right) \quad ; \quad L_{2}^{*}=\left(\begin{array}{ll}1 & 1\end{array}\right)\left[\begin{array}{ll}M_{3}^{*}+\left(\begin{array}{ll}M_{1} & M_{2}\end{array}\right)-G_{2}\end{array}\right]\left(\begin{array}{cc}-\mathbb{I} & 0 \\ 0 & \mathbb{I}\end{array}\right)$

This finally gives when used in (5), keeping only first order terms:

$\left(\frac{1}{4} m_{1} l_{1}^{2}+\frac{1}{2} m_{1} l_{1}\left(l_{3}+l_{4}\right)+I_{1}\right) \ddot{q}_{1}=\frac{1}{2} m_{1} l_{1} \ddot{x}+\frac{1}{2} m_{1} l_{1} g q_{1}-\left(L_{0}^{*} X_{c}+L_{2}^{*} \ddot{X}_{C}\right)$

According to (12), $\ddot{x}$ can be written as:

$$
\ddot{x}=\left(\begin{array}{llll}
-\left(l_{3}+l_{4}\right) & 0 & -l_{4} & 0
\end{array}\right)\left(\begin{array}{cc}
-\mathbb{I} & 0 \\
0 & \mathbb{I}
\end{array}\right) \ddot{X}_{c}=: D \ddot{X}_{c}
$$

therefore,

$$
\ddot{q}_{1}=\frac{\frac{1}{2} m_{1} l_{1} g}{d} q_{1}-\frac{L_{0}^{*}}{d} X_{c}+\frac{\frac{1}{2} m_{1} l_{1} D-L_{2}^{*}}{d} \ddot{X}_{c}
$$

where $d=\frac{1}{4} m_{1} l_{1}^{2}+\frac{1}{2} m_{1} l_{1}\left(l_{3}+l_{4}\right)+I_{1}$. (19) writes:

$$
\ddot{q}_{1}=a q_{1}-L_{0} X_{c}+L_{2} \ddot{X}_{c}
$$

\section{A. Generation of reference trajectories for $X_{c}$}

The reference trajectories of $X_{c}$ on the time interval $\left[t_{0}, t_{f}\right]$ are completely parameterized by the boundary conditions $X_{c}\left(t_{0}\right), \dot{X}_{c}\left(t_{0}\right), X_{c}\left(t_{f}\right), \dot{X}_{c}\left(t_{f}\right)$. For instance, the reference trajectories on each of the scalar variables $q_{31}, q_{32}, q_{41}$ and $q_{42}$ is the unique $3^{\text {rd }}$ order polynomial satisfying the four boundary conditions. This can be written in a compact form as follows:

$$
\begin{aligned}
& X_{c}(t)=\Phi(t) \Psi \mathscr{X} \\
& \mathscr{X}=\left(X_{c}\left(t_{0}\right) \quad \dot{X}_{c}\left(t_{0}\right) \quad X_{c}\left(t_{f}\right) \quad \dot{X}_{c}\left(t_{f}\right)\right)^{T} \in \mathbb{R}^{16} \\
& \Phi(t):=\left(\begin{array}{cccc}
\varphi(t) & 0 & 0 & 0 \\
0 & \varphi(t) & 0 & 0 \\
0 & 0 & \varphi(t) & 0 \\
0 & 0 & 0 & \varphi(t)
\end{array}\right) \in \mathbb{R}^{4 \times 16} \\
& \varphi(t)=\left(\begin{array}{llll}
1 & t & t^{2} / 2 & t^{3} / 6
\end{array}\right) \\
& \Psi=\left(\begin{array}{l}
\Phi\left(t_{0}\right) \\
\dot{\Phi}\left(t_{0}\right) \\
\Phi\left(t_{f}\right) \\
\dot{\Phi}\left(t_{f}\right)
\end{array}\right)^{-1} \quad \in \mathbb{R}^{16 \times 16}
\end{aligned}
$$

\section{B. Evolution of unactuated coordinate under perfect tracking}

Let us assume that the reference trajectories on $X_{c}$ defined above are perfectly tracked. This is not really a restrictive assumption since $X_{c}$ is totally controlled by the four control inputs $u \in \mathbb{R}^{4}$. The only obstacle upon such a perfect tracking comes from either saturations or insufficient contact forces. However, the class of small angles trajectories clearly disposes of such problems. For the time being, let us concentrate on what happens to the torso's angle under perfect tracking. Injecting (21) in (20) gives:

$$
\ddot{q}_{1}=a q_{1}-\left(L_{0} \Phi(t)+L_{2} \ddot{\Phi}(t)\right) \Psi \mathscr{X}
$$

Let us define the state of the torso by $\Theta:=\left(q_{1} \dot{q}_{1}\right)^{T}$, using (23), one may write:

$$
\begin{aligned}
\dot{\Theta}(t) & =\left(\begin{array}{ll}
0 & 1 \\
a & 0
\end{array}\right) \Theta(t)+\left(\begin{array}{c}
0_{1 \times 16} \\
-\left(L_{0} \Phi(t)+L_{2} \ddot{\Phi}(t)\right) \Psi
\end{array}\right) \mathscr{X} \\
& =: A \Theta(t)+B(t) \mathscr{X}
\end{aligned}
$$

Therefore, one clearly has:

$$
\begin{gathered}
\Theta\left(t_{f}\right)=\bar{A}\left(t_{0}, t_{f}\right) \Theta\left(t_{0}\right)+\bar{B}\left(t_{0}, t_{f}\right) \mathscr{X} \\
\bar{A}\left(t_{0}, t_{f}\right)=\bar{A}\left(0, t_{f}-t_{0}\right):=\exp \left[A\left(t_{f}-t_{0}\right)\right] \\
\bar{B}\left(t_{0}, t_{f}\right)=\bar{B}\left(0, t_{f}-t_{0}\right):=\int_{t_{0}}^{t_{f}} \exp \left(A\left(t_{f}-\tau\right)\right) B(\tau) d \tau
\end{gathered}
$$

\section{Recall on impact's dynamics}

In this section, the principle of the impact dynamics used in the forthcoming developments is recalled. For that, consider first the expressions of the feet cartesian coordinates $x_{p_{i}}(q), y_{p_{i}}(q)$. Let us then define $J_{i}(q)$ (for $i \in\{1,2\}$ ) as follows:

$$
J_{i}(q):=\frac{\partial}{\partial q}\left(\begin{array}{l}
y_{p_{i}}(q) \\
x_{p_{i}}(q)
\end{array}\right) \in \mathbb{R}^{2 \times 7}
$$

Let us define also (for $i \in\{1,2\}$ ) the Lagrange multipliers relative to the contact forces by $\lambda_{i}=\left(\begin{array}{l}\lambda_{n i} \\ \lambda_{t i}\end{array}\right)\left(\lambda_{n i}\right.$ : relative to the normal force of the foot $i$, and $\lambda_{t i}$ : relative to its tangential force). The single impact dynamics can then be given by:

$$
\begin{aligned}
& M(q)\left(\dot{q}^{+}-\dot{q}^{-}\right)=J_{2}^{T}(q) \lambda_{2} \\
& J_{2}(q) \dot{q}^{+}=0 \\
& J_{1}(q) \dot{q}^{+} \geq 0 \\
& \Lambda\left(\mu_{0}\right) \lambda_{2} \geq 0
\end{aligned}
$$

$\checkmark$ Equation (30) states that the formerly swing leg is becoming the new support one, without rebound nor slip at impact.

$\checkmark$ Inequality (31) states that the formerly stance leg is now leaving the ground.

$\checkmark$ Inequality (32) guarantees the classical non penetration and non slipping conditions at the contact point. 
$\checkmark$ The matrix $\Lambda\left(\mu_{0}\right)$ used in the last inequality is given by

$$
\Lambda\left(\mu_{0}\right):=\left(\begin{array}{cc}
1 & 0 \\
\mu_{0} & 1 \\
\mu_{0} & -1
\end{array}\right) \in \mathbb{R}^{3 \times 2}
$$

The solution of (29)-(30) is given by (3). Let:

$$
\begin{aligned}
Q_{1}(q) & :=\left[I-M^{-1} J_{2}^{T}\left(J_{2} M^{-1} J_{2}^{T}\right)^{-1} J_{2}\right] \\
Q_{2}(q) & =\left[\left(J_{2} M^{-1} J_{2}^{T}\right)^{-1} J_{2}\right] \\
Q_{3}(q) & =J_{1}(q) Q_{1}(q) \\
Q_{4}(q) & =\Lambda\left(\mu_{0}\right) Q_{2}(q)
\end{aligned}
$$

then the single support impact constraints resume to:

$$
\begin{gathered}
\dot{q}^{+}=Q_{1}(q) \dot{q}^{-} \\
Q_{3}(q) \dot{q}^{-} \geq 0 \\
Q_{4}(q) \dot{q}^{-} \geq 0
\end{gathered}
$$

Note that (35)-(36) insure a well-defined single support-type impact, while equation (34) gives the velocities post-impact function of positions and velocities pre-impact.

\section{FEEDBACK DEFINITION}

The starting point of the proposed feedback is the torso's dynamic (20). Since $X_{c} \in \mathbb{R}^{4}$ is completely controllable via existing actuators, one may legitimately discuss on the basis of the following 10-dimensional controllable system

$$
\begin{aligned}
& \ddot{q}_{1}=a q_{1}-L_{0} X_{c}+L_{2} v \\
& \ddot{X}_{c}=v
\end{aligned}
$$

In the following, the choice of the alternative control $v \in \mathbb{R}^{4}$ will be explained. Equations (37)-(38) can be easily put in a state-space form:

$$
\dot{Z}=A Z+B v
$$

$Z:=\left(\begin{array}{l}q_{1} \\ X_{c} \\ \dot{q}_{1} \\ \dot{X}_{c}\end{array}\right) \in \mathbb{R}^{10} ; A:=\left(\begin{array}{cccc}0 & 0 & 1 & 0 \\ 0 & 0 & 0 & \mathbb{I} \\ a & -L_{0} & 0 & 0 \\ 0 & 0 & 0 & 0\end{array}\right) ; B:=\left(\begin{array}{c}0 \\ 0 \\ L_{2} \\ \mathbb{I}\end{array}\right)$

where the 0 elements in (40) are null matrices of suitable dimensions and $\mathbb{I}$ is the identity matrix in $\mathbb{R}^{4 \times 4}$. Since (39) is completely controllable, the only thing to do is to define a final state $Z\left(t_{f}\right)$ that is to be reached at $t=t_{f}$ together with a transient quadratic cost function to be minimized.

Consider the following notations:

$Z=\left(\begin{array}{c}Z_{1} \\ Z_{2}\end{array}\right) \quad ; \quad Z_{1}:=\left(\begin{array}{c}q_{1} \\ X_{c}\end{array}\right) \in \mathbb{R}^{5} \quad ; \quad Z_{2}:=\left(\begin{array}{c}\dot{q}_{1} \\ \dot{X}_{c}\end{array}\right) \in \mathbb{R}^{5}$

Let $q=K\left(Z_{1}\right)$ be the function that gives $q$ under swing phase assumption on the feet 1 , let us also define $K^{\prime}\left(Z_{1}\right):=$ $\frac{\partial K}{\partial Z_{1}}\left(Z_{1}\right)$. Finally for all $q \in \mathbb{R}^{7}, \Pi_{i} q$ refers to the $i^{t h}$ component of $q$. The final state $Z^{f}=\left(Z_{1}^{f^{T}} Z_{2}^{f^{T}}\right)^{T}$ is therefore defined as follows:
1) First, the position vector $Z_{1}^{f}$ is a priori given. it must reflect the desired mean velocity of the walk. Furthermore, it must respect the basic "small angle" assumption. All the following rules aim to define $Z_{2}^{f}$.

2) The torso's angular velocity just after the impact must be 0 :

$$
\left[\Pi_{5} Q_{1}\left(K\left(Z_{1}^{f}\right)\right) K^{\prime}\left(Z_{1}^{f}\right)\right] Z_{2}^{f}=0
$$

3) The horizontal hip's velocity $\dot{x}$ just after the impact must be equal to $v^{r}$, this can be written as follows:

$$
\left[\Pi_{6} Q_{1}\left(K\left(Z_{1}^{f}\right)\right) K^{\prime}\left(Z_{1}^{f}\right)\right] Z_{2}^{f}=v^{r}
$$

4) The vertical hip's velocity $\dot{y}$ just after the impact must be equal to 0 , this can be written as follows

$$
\left[\Pi_{7} Q_{1}\left(K\left(Z_{1}^{f}\right)\right) K^{\prime}\left(Z_{1}^{f}\right)\right] Z_{2}^{f}=0
$$

5) The vertical velocity of the free foot just before the impact must be equal to some $v^{p}<0$ while its horizontal velocity must be 0 , that is:

$$
\left[J_{2}\left(K\left(Z_{1}^{f}\right)\right) K^{\prime}\left(Z_{1}^{f}\right)\right] Z_{2}^{f}=\left(\begin{array}{c}
v_{p} \\
0
\end{array}\right)
$$

6) The impact inequalities (35)-(36) must be satisfied:

$$
\begin{aligned}
& {\left[Q_{3}\left(K\left(Z_{1}^{f}\right)\right) K^{\prime}\left(Z_{1}^{f}\right)\right] Z_{2}^{f} \geq 0} \\
& {\left[Q_{4}\left(K\left(Z_{1}^{f}\right)\right) K^{\prime}\left(Z_{1}^{f}\right)\right] Z_{2}^{f} \geq 0}
\end{aligned}
$$

7) The final constraint concerns the contact conditions on the trajectory joining the final state $Z^{f}$. To express this condition, note that under the "small angles" assumption, equations (9)-(10) together with (18) can be invoked to argue that $\lambda_{n} \geq 0$ is obviously satisfied and that the condition $\left|\lambda_{t}\right| \leq \mu_{0} \lambda_{n}$ resumes to:

$$
-\alpha \mu_{0} m g \leq \bar{D} v \leq \alpha \mu_{0} m g
$$

where

$$
\left.\bar{D}=\left[\begin{array}{llll}
\frac{g_{34}}{g} & \frac{g_{34}}{g} & \frac{g_{4}}{g} & \frac{g_{4}}{g}
\end{array}\right)\left(\begin{array}{cc}
-\mathbb{I} & 0 \\
0 & \mathbb{I}
\end{array}\right)-m D\right]
$$

$\alpha \in] 0,1[$ is a security margin. Note that (48) is a saturation condition on the control $v$ of the system (39) under interest.

These constraints can be summarized in:

$A_{e q}\left(Z_{1}^{f}\right) Z_{2}^{f}=b_{e q}\left(v_{r}, v_{p}\right) ; A_{\text {ineq }}\left(Z_{1}^{f}\right) Z_{2}^{f} \geq 0 ; A_{s} v \leq b_{s}$ 


$$
\begin{aligned}
A_{e q}\left(Z_{1}^{f}\right):=\left(\begin{array}{c}
\Pi_{5} Q_{1}\left(K\left(Z_{1}^{f}\right)\right) K^{\prime}\left(Z_{1}^{f}\right) \\
\Pi_{6} Q_{1}\left(K\left(Z_{1}^{f}\right)\right) \\
\Pi_{7} Q_{1}\left(K\left(Z_{1}^{f}\right)\right) \\
J_{2}\left(K\left(Z_{1}^{f}\right)\right) \\
K^{\prime}\left(Z_{1}^{f}\right)
\end{array}\right) \in \mathbb{R}^{5 \times 5} ; \quad b_{s}=\alpha \mu_{0} m g\left(\begin{array}{l}
1 \\
1
\end{array}\right) \\
b_{\text {eq }}\left(v_{r}, v_{p}\right):=\left(\begin{array}{c}
0 \\
v^{r} \\
0 \\
v^{p} \\
0
\end{array}\right) ; \quad A_{\text {ineq }}\left(Z_{1}^{f}\right):=\left(\begin{array}{c}
Q_{3}\left(K\left(Z_{1}^{f}\right)\right) K^{\prime}\left(Z_{1}^{f}\right) \\
Q_{4}\left(K\left(Z_{1}^{f}\right)\right) K^{\prime}\left(Z_{1}^{f}\right)
\end{array}\right) ; \quad A_{s}:=\left(\begin{array}{c}
\bar{D} \\
-\bar{D}
\end{array}\right)
\end{aligned}
$$

Therefore, the desired final state $Z_{f}$ is now uniquely determined by the choice of $Z_{1}^{f}$ as follows

$$
\hat{Z}\left(Z_{1}^{f}\right):=\left(\begin{array}{c}
Z_{1}^{f} \\
\hat{Z}_{2}^{f}\left(Z_{1}^{f}, v_{r}, v_{p}\right)
\end{array}\right)
$$

where $\hat{Z}_{2}^{f}$ is solution of the following linearly constrained quadratic optimization problem:

$\hat{Z}_{2}^{f}\left(Z_{1}^{f}, v_{r}, v_{p}\right):=\operatorname{Arg} \min _{Z_{2}^{f} \in \mathscr{L}}\left\{\left\|A_{e q}\left(Z_{1}^{f}\right) Z_{2}^{f}-b_{e q}\left(v_{r}, v_{p}\right)\right\|^{2} \quad\right.$ under $\left.\quad A_{\text {ineq }}\left(Z_{1}^{f}\right) Z_{2}^{f} \geq 0\right\}$

where $\mathscr{Z} \subset \mathbb{R}^{5}$ is the set of admissible values reflecting the "small angles" assumption.

With the final state $Z^{f}$ in hand, the problem is now to define a state feedback to be applied during the step over the interval $\left[0, t_{f}\right]$ so that $Z\left(t_{f}\right)=Z^{f}$. For, let us give some useful notations

- Assume that a discret-time scheme is used with a sampling time of $\tau>0$.

- Let $\Phi_{i}$ and $\Psi_{i}$ be the $i$-steps discrete transition matrices for the linear system (39), namely

$$
Z(i \tau)=\Phi_{i} Z(0)+\Psi_{i} \tilde{v} \quad ; \quad \tilde{v} \in \mathbb{R}^{4 \times i}
$$

- The walking step duration $t_{f}$ is a multiple of the sampling time $\tau$, namely $t_{f}=N \cdot \tau$.

Since a finite time response is required (for $Z$ to reach $Z^{f}$ ), time-varying feedback control is necessary. For that, let $k \in$ $\{0,1, \ldots, N-1\}$ represent the discrete time counter during the walking step time-interval. Therefore, at each sampling instant $k \in\{0,1, \ldots, N-1\}$ one has to solve the following linearly constrained quadratic problem:

$$
\begin{aligned}
& \hat{v}\left(k, Z(k), Z^{f}\right):=\quad \begin{array}{ll}
\operatorname{Arg} \min _{\tilde{v} \in \mathbb{R}^{4(N-k)}}\left\|\Psi_{N-k} \tilde{v}+\Phi_{N-k} Z(i)-Z^{f}\right\|^{2} \\
\text { under } \tilde{A}_{s} \tilde{v} \leq \tilde{b}_{s}
\end{array} \\
& \tilde{A}_{s}:=\left(\begin{array}{ccccc}
A_{s} & 0 & \ldots & 0 & 0 \\
0 & A_{s} & 0 & \ldots & 0 \\
\ldots & \ldots & \ldots & \ldots & \ldots \\
\ldots & \ldots & \ldots & \ldots & \ldots \\
0 & 0 & \ldots & 0 & A_{s}
\end{array}\right) \in \mathbb{R}^{2(N-k) \times 4(N-k)} \\
& \tilde{b}_{s}:=\left(\begin{array}{c}
b_{s} \\
\vdots \\
b_{s}
\end{array}\right) \in \mathbb{R}^{2(N-k)}
\end{aligned}
$$

\section{NUMERICAL SIMULATIONS}

The objective of this simulation scenario is the application of the proposed strategy to control the biped robot RABBIT for a dynamic walking gait starting from rest. The walking-step duration is $t_{f}=1.2 \mathrm{sec}$, the desired foot preimpact velocity is $v_{p}=-0.07 \mathrm{~m} / \mathrm{sec}$. Initial condition on the unactuated coordinate is $\left(q_{1}, \dot{q}_{1}\right)_{0}=\left(0,6^{\circ}\right)$, the sampling time is $\tau=0.05 \mathrm{sec}$, and the mean walking velocity is $v_{r}=-0.132 \mathrm{~m} / \mathrm{sec}$. The obtained simulation results are plotted in figures 3-7. Figure 3 displays the evolution of the articular positions and velocities of the robot's femurs versus time. Figure 4 depicts the evolution of the articular positions and velocities of the robot's tibias versus time. From these figures concerning the actuated coordinates (direct controlled variables) of the robot, it can be seen clearly the convergence to a cyclic walking gait. The evolution of the unactuated coordinate is plotted in figure 5 which represents the articular position and velocity of the torso versus time, where it (5ean also be seen the convergence a cyclic trajectory. Figure 6 shows the evolution of the y-cartesian hip's trajectories versus time. The control inputs, are depicted in figure 7, showing the torques to be generated by the actuators.

\section{CONCLUSION}

In this paper a discret-time control strategy is proposed to control a five-link four-actuator biped walking robot. The proposed control strategy consists in a choice of a particular configurations that enables a full state controllability avoiding the need to use Poincaré-like argumentation in the proof of convergence to stable cyclic walking gaits. The basic idea relies in considering small angles, which enables us to express the dynamics of the indirectly controlled variable (unactuated coordinate) as linear function of the directly controlled variables (actuated coordinates) and their derivatives. Then a discrete-time feedback is proposed to control the whole system in the aim to reproduce a cyclic waling gait. A demonstration movie accompanying the paper shows the obtained walking gait.

\section{REFERENCES}

[1] L. Beck. Most famous robots (the humanoid robot). http://sites.google.com/site/luisbeck007/humanoid, 2009.

[2] N. Chaillet, G. Abba, and E. Ostertag. Double dynamic modelling and computed torque control of a biped robot. In Proc. IEEE/RSJ Int. Conf. Intel. Robotics Systems., pages 1149-1153, Munich , Germany, 1994.

[3] T. Chang and Y. Hurmuzlu. Sliding control without reaching phase and its application to bipedal locomotion. Journal of Dynamics Systems, Measurement and Control, 115:447-455, 1993.

[4] A. Chemori and M. Alamir. Multi-step limit cycle generation for rabbit's walking based on a nonlinear low dimensional predictive control scheme. Mechatronics, Elsevier, 16(5):259-277, 2006.

[5] A. Chemori and A. Loria. Control of a planar under-actuated biped on a complete walking cycle. IEEE Trans. on Automat. Contr., 49(5):838843, May 2004.

[6] C. Chevallereau G. Abba Y. Aoustin F. Plestan E.R. Westervelt C. Canudas de Wit and J.W. Grizzle. Rabbit: A testbed for advanced control theory. IEEE Control Systems Magazine, 23(5):57- 79, August 2003.

[7] J. W. Grizzle, G. Abba, and F. Plestan. Asymptotically stable walking for biped robots: analysis via systems with impulse effects. IEEE Trans. on Automat. Contr., 46(1):51-64, January 2001. 

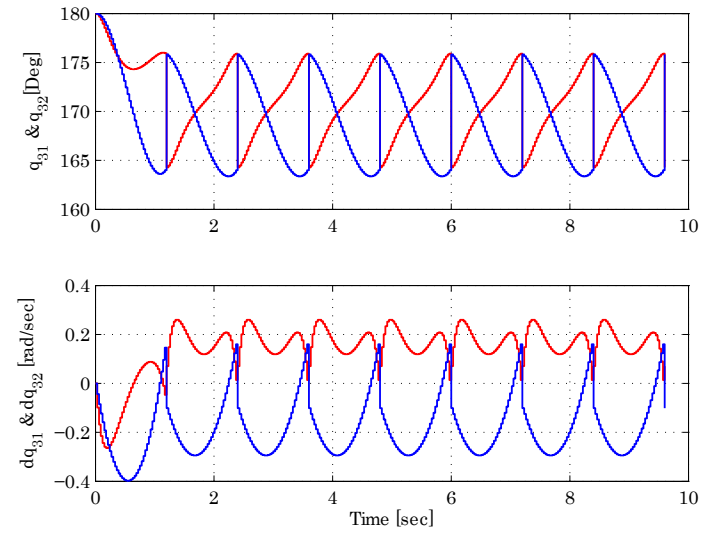

Fig. 3. Evolution of the femur's positions and velocities versus time
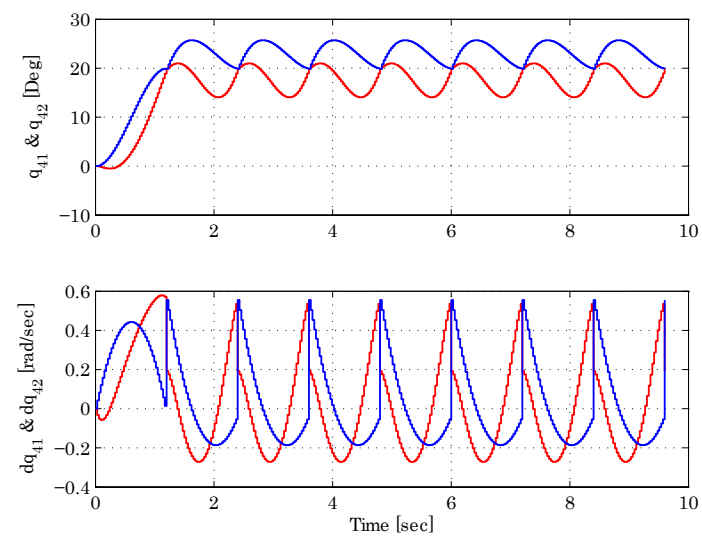

Fig. 4. Evolution of the tibia's positions and velocities versus time

[8] M. Hardt, K. Kreutz-Delgado, and J. Helton. Minimal energy control of a biped robot with numerical methods and a recursive symbolic dynamic model. In Proc. 37th. IEEE Conf. Decision Contr., pages 413-416, Florida, USA, 1998.

[9] Y. Hurmuzlu and D.B. Marghitu. Rigid body collisions of planar kinematic chains with multiple contact points. Int. J. Rob. Research, 13(1):82-92, 1994.

[10] A. Kun and W. Miller. Adaptive dynamic balance of an experimental biped robot. In Proc. IEEE Conf. Robotics Automat., 1996.

[11] L. Magdalena and F. Monasterio-Huelin. A fuzzy logic controller with learning through the evolution of its knowledge base. International Journal of Approximate Reasoning, 16(3/4):335-358, 1997.

[12] J. H. Park. Impedance control for biped robot locomotion. IEEE Trans. on Robotics Automat., 17(6):870-882, December 2001.

[13] J. Pratt and G. Pratt. Intuitive control of a planar bipedal walking robot. In Proc. IEEE Conf. Robotics Automat., pages 2014-2021, Leuven, Belgium, 1998.

[14] M. H. Raibert. Legged robots. Commmun. ACM, 29:499-514, 1986.

[15] L. Roussel, C. Canudas, and A. Goswami. Generation of energy optimal complete gait cycles for biped robots. In Proc. IEEE Conf. Robotics Automat., pages 2036-2041, Leuven, Belgium, 1998.

[16] M. Spong. Passivity based control of the compass gait biped. In IFAC World Congress, Beijing, China, 1999.

[17] M. Spong and M. Vidyasagar. Robot Dynamics and Control. John Wiley \& Sons, New York, 1989.

[18] Y. F. Zheng and H. Hemami. Impact effects of biped contact with the environment. IEEE Trans. on Syst., Man and Cyb, 14(3):51-64, May/June 1984.
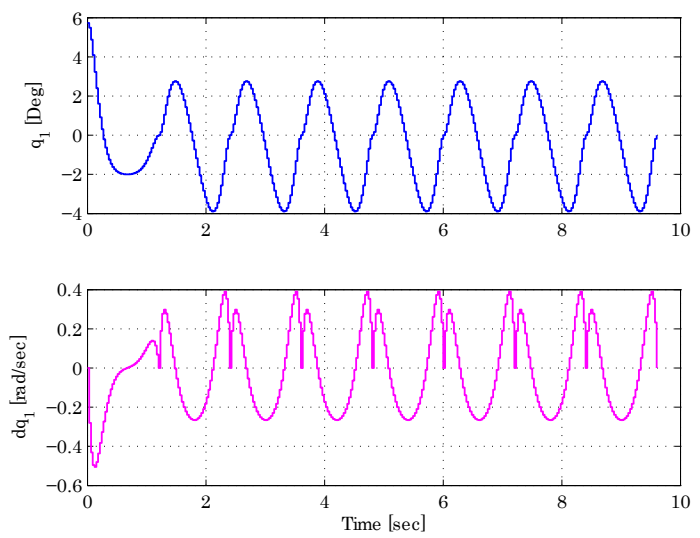

Fig. 5. Evolution of the torso's (unactuated coordinate) position and velocity versus time
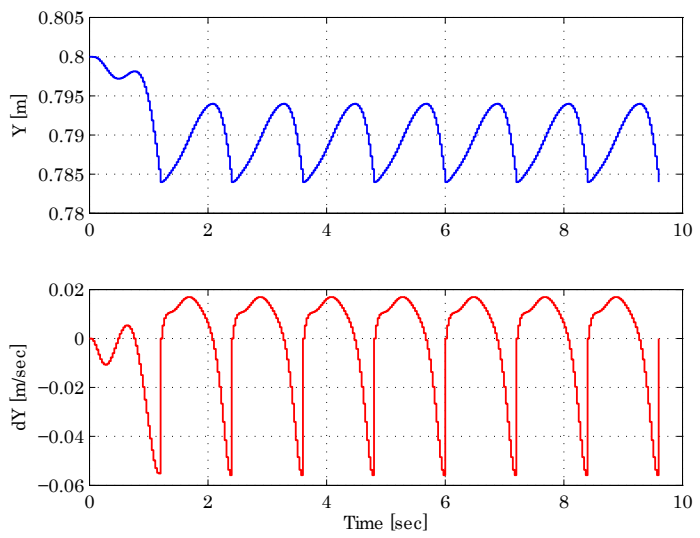

Fig. 6. Evolution of the hip's $y$-cartesian position and velocity
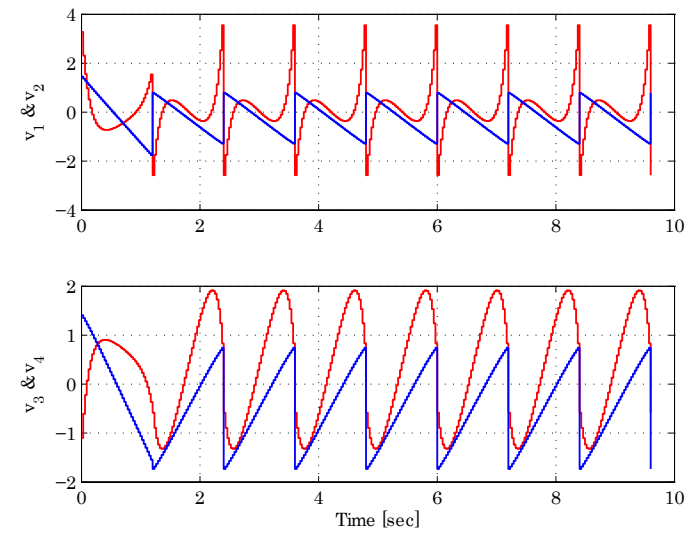

Fig. 7. Evolution of the control inputs versus time 